\title{
DisTANCIA SEMÁNTICA Y NEOLÓGICA LÉXICA *
}

\author{
Constanza Gerding Salas** \\ Gabriela Kotz Grabole***
}

\section{Resumen}

Los neologismos semánticos, difíciles de estudiar a nivel lexicológico y lexicográfico, son abordados en este estudio en su forma nominal en español y desde la perspectiva de la Estructura de Qualia, en que la polisemia se explica según los atributos del referente. Se plantea una adecuación de la tipologización existente en función de la distancia semántica entre el sentido establecido y el nuevo para una mejor clasificación de las creaciones léxicas con base en el contenido. Los criterios de tipificación fueron la información compartida entre el sentido establecido y el nuevo, la naturaleza del cambio semántico y la configuración del significado. Este modelo resultó aplicable al corpus, y la propuesta, con una nueva gradación de la distancia semántica entre ambos sentidos, facilitó la clasificación de los neologismos semánticos.

Palabras clave: neologismo semántico, Teoría del Lexicón Generativo, Estructura de Qualia, distancia semántica.

\section{SEMANTIC DISTANCE AND LEXICAL NEOLOGY}

\begin{abstract}
Semantic neologisms are difficult to study both lexicologically and lexicographically. This paper discusses new semantic nouns in Spanish from the perspective of the Qualia Structure, which explains polysemy through the attributes of referents. An adaptation of the existing typology is proposed using the semantic distance between the established meaning and the new one in order to accomplish a better classification of semantic neologisms on the basis of content. The classification criteria were the shared information between the established meaning and the new one, the nature of the semantic change, and the neological configuration of meaning. This model proved to be applicable to the corpus, and the new gradation of the semantic distance between both meanings made the classification of semantic neologisms easier.
\end{abstract}

Keywords: semantic neologism, Generative Lexicon Theory, Qualia Structure, semantic distance.

Recibido: 19-05-2015

Aceptado: 20-11-2015

* Este trabajo se inscribe en el Proyecto de Investigación liderado por el Observatorio de Neología del Institut Universitari de Lingüistica Aplicada de la Universidad Pompeu Fabra, Barcelona. En Chile el Proyecto Antenas Neológicas lo desarrolla la Antena Chilena de Neología del Grupo de Estudios Terminológicos, código 03.F5.01de la Dirección de Investigación de la Universidad de Concepción.

** Chilena. Doctora en Educación de la Universidad de Estocolmo y la Universidad de Concepción. Académica de la Universidad de Concepción, Concepción, Chile. cgerding@udec.cl

*** Argentina. Doctora en Lingüística de la Universidad de Concepción. Académica de la Universidad de Concepción, Concepción, Chile.gkotz@udec.cl 


\section{Introducción}

En el presente estudio se analiza la neología semántica nominal en español a partir de la Teoría del Lexicón Generativo formulada por Pustejovsky $(1991,1995,2011)$ y se propone comprobar la aplicabilidad de la clasificación de los neologismos semánticos en el corpus de la Antena Chilena de Neología sobre la base de la Teoría de Qualia (De Miguel, 2009; Adelstein, 2011; Álvarez de Mon Rego \& Álvarez-Bolado Sánchez, 2013; Berri, 2014). Una descripción más rigurosa de los cambios semánticos permitiría una tipologización más precisa de las piezas léxicas con el propósito de mejorar la elaboración lexicográfica (Berri \& Adelstein, 2010). En esta actividad se suelen producir conflictos entre el 'sentimiento de neologicidad' (Sablayrolles, 2000) y el criterio lexicográfico empleados para la detección, el análisis y la clasificación de neologismos. Y esta propuesta pretende contribuir a despejar este dilema. En esta investigación, el análisis se circunscribe al sustantivo por su mayor representatividad en los corpus neológicos y en los estudios de neología semántica (De Miguel, 2009; Adelstein, 2011; Álvarez de Mon Rego \& Álvarez-Bolado Sánchez, 2013; Berri, 2014).

Las nuevas tendencias de la investigación en creación léxica se basan en el contenido, lo que se ha visto reflejado en estudios realizados por investigadores vinculados a la Red Antenas Neológicas (Berri \& Adelstein, 2010; Adelstein, 2011), entidad que surge al alero del Observatori de Neología del Institut Universitari de Lingüística Aplicada de la Universitat Pompeu Fabra (OBNEO), que reúne a grupos de investigación en las variantes del español de Argentina, Cataluña, Chile, Colombia, México y Perú. La Red Antenas Neológicas adscribe a la propuesta general de clasificación de neología léxica de Cabré (2006), que consta de las siguientes macrocategorías: neologismos formales, neologismos formados por variación, neologismos sintagmáticos, neologismos semánticos, préstamos con y sin adaptación y una categoría 'otros' para piezas léxicas difíciles de etiquetar. De esta tipología, a la Antena Chilena de Neología le han llamado especialmente la atención los neologismos semánticos por lo peculiares y complejos que resultan para el neólogo a la hora de desentrañar el nuevo sentido.

Tradicionalmente, los neologismos semánticos se han explicado mediante mecanismos de analogía, ampliación, restricción o sustitución 
de los rasgos semánticos de una unidad existente (Cabré, Bayà, Bernal, Freixá, Solé \& Vallès, 2002) o bien mediante metáforas o metonimias respecto del sentido original (Guerrero Ramos, 2010) y, sobre esa base, se han recopilado y estudiado como acepciones completa o parcialmente neológicas.

Para identificar estas nuevas acepciones, el criterio primario es el psicolingüístico (Rey, 1976), que se refleja en el 'sentimiento de neologicidad' (Sablayrolles, 2000), es decir, aquella intuición que tienen los hablantes acerca de las palabras nuevas en su propia lengua. Luego de este primer acercamiento, en la Red Antenas Neológicas se sanciona el carácter neológico de una pieza léxica mediante el criterio lexicográfico que, si bien adolece de algunas limitaciones, es más objetivable y es congruente con una de las metas del OBNEO (2003), cual es la de contribuir a la actualización de diccionarios de lengua general. Este criterio consiste en la comprobación de la presencia de las piezas léxicas en un corpus lexicográfico de exclusión preestablecido (Cabré \& Estopà, 2009). Sin embargo, en el caso de la neología semántica, no basta con el cotejo de las unidades léxicas con las de una batería de diccionarios, porque el criterio lexicográfico no es satisfactorio para establecer la configuración semántica que permita zanjar el nivel de neologicidad de las piezas léxicas (Adelstein, 2011).

Según el Protocolo de vaciado de prensa escrita delOBNEO (2003), por el cual se rige la Red Antenas Neológicas, la recopilación de neologismos semánticos se ciñe a dos criterios generales:

Según el criterio 1, se consideran neologismos semánticos las piezas léxicas formadas a partir de una modificación del significado de una base léxica existente. Ejemplo:

- En el campamento "Nueva Aurora", los pobladores tienen dolor y rabia, porque confiaron y tuvieron la esperanza de que por fin recibirían una casa digna. [El Sur, Chile, 1/04/2011]

El carácter neológico semántico del primer ejemplo está dado por una modificación del significado de la acepción existente en el Diccionario de la lengua española (Real Academia Española, 2001), donde la unidad 'campamento', en su acepción 2, se define como "Instalación eventual, en terreno abierto, de personas que van de camino o que se reúnen para un fin especial". En la unidad recogida en la variante chilena, 
en cambio, se refiere a un 'barrio marginal con grandes carencias de infraestructura y con viviendas de calidad muy precaria y de reducido tamaño, que emerge de forma espontánea y paulatina por una necesidad habitacional de personas de escasos recursos económicos'. Esta unidad léxica es equivalente sinónimo de 'villa miseria', unidad registrada para la variante argentina del español en el corpus lexicográfico de exclusión ${ }^{1}$ del OBNEO.

Según el criterio 2, se consideran neologismos semánticos las piezas léxicas formadas a partir de un nombre propio de persona o de marca registrada utilizado como nombre común, es decir, como resultado de una metonimia o bien por antonomasia (Cabré, 2006). Ejemplo:

- En Japón, y sobre todo en los hoteles, existe la práctica del "toilegami" (origami de confort) que muestra un doblado artístico del extremo del papel higiénico y que indica a los visitantes que el papel está virgen o que el lugar ha sido higienizado. [Las Últimas Noticias, Chile, 30/06/2013]

En el ejemplo precedente se hace referencia a la palabra con la que popularmente se denomina al papel sanitario en Chile, que corresponde a un uso metonímico de la marca registrada de papel higiénico 'Confort'.

Sin duda, la neología semántica, por su naturaleza, ofrece gran dificultad para su detección y análisis, ya que no presenta evidencia formal de modificación del sentido original (Cabré, 2006). La principal dificultad para determinar la neologicidad de las unidades léxicas semánticas radica en que la ampliación o la restricción del sentido no se alejan demasiado del sentido original o, quizás, en que el nuevo uso es completamente metafórico. De allí, entonces, la importancia del contexto para la identificación y posterior caracterización de las nuevas piezas léxicas.

Tradicionalmente, se establece que el contexto lingüístico o 'cotexto' está formado por el material lingüístico que precede y sigue a un enunciado. En un acto de comunicación verbal, el cotexto adquiere gran importancia al momento de inferir el significado de enunciados

1 El corpus lexicográfico de exclusión del OBNEO está constituido por el Diccionario de la lengua española de la Real Academia Española de la Lengua, 2001. 22a edición. Madrid: Espasa Calpe. Disponible en http:/ / www.rae.es y por el Diccionario de uso del español de América y España, 2002. Vox, Madrid. 
desconocidos. En términos amplios, el 'contexto' es el entorno lingüístico, social y pragmático que rodea a una palabra, frase u oración y que condiciona, modificando o no, el significado de ésta (Coșeriu, 1973; Miller, Beckwith, Fellbaum, Gross \& Miller, 1990).

La detección de neologismos semánticos es posible mediante la 'actualización' u "operación interpretativa que permite identificar o construir un sema en contexto" (Pequeño glosario de semántica, 2014); a través de esta misma operación, conjuntamente con el conocimiento de mundo del hablante, se puede desentrañar el nuevo sentido de una pieza léxica. Cuando un contexto permite que se actualice uno o más sentidos de una pieza léxica, se está en presencia de 'ambigüedad inclusiva' (Berri, 2014) y, en tal caso, la desambiguación se podría conseguir observando un contexto más amplio o considerando elementos extralingüísticos. En efecto, la activación de una pieza léxica semántica en un contexto distinto al habitual es un indicio de modificación de su significado, ya que se observa un cambio en el perfil de concurrencia (Nazar \& Vidal, 2008).

Por otra parte, en su modelo del Lexicón Generativo, Pustejovsky (1991, 1995, 2011) explica la polisemia sobre la base del contexto y el uso creativo de las palabras. Este modelo permite la representación de la estructura multidimensional del significado y la clasificación de categorías léxicas en tipos semánticos. Para Pustejovsky (1991) la descomposición léxica se puede realizar de manera generativa y es necesaria una apreciación de la estructura sintáctica para el estudio de la semántica léxica, ya que el significado no puede estar completamente disociado de la estructura que lo porta. La convicción de Pustejovsky (1991) es que la representación del contexto de un enunciado involucra muchos factores generativos diferentes que develan la forma en que los hablantes crean y manipulan el contexto para que sea entendido.

Este modelo propone una estructura semántica constituida por cuatro niveles de representación que conforman el Lexicón Generativo: a) una estructura argumental, que especifica el número y la naturaleza de los argumentos del predicado y el modo en que se realizan; $b$ ) una estructura eventiva, que especifica el tipo de evento denotado por su predicado y sus estructuras subeventivas; c) una estructura de tipificación léxica, que explica la forma en que se relaciona una palabra con otras del lexicón, y d) una estructura de qualia, que codifica formalmente las relaciones lógicas en distintas dimensiones. 
La Teoría de la Estructura de Qualia que desarrolló Pustejovsky (1991) es como un lenguaje de representación de los atributos del referente de un ítem léxico con el que se explica la polisemia regular o polisemia sistemática de las palabras (De Miguel, 2009; Berri \& Adelstein, 2010; Adelstein, 2011):

a) En el quale formal se ofrece información sobre lo que distingue a la entidad designada en un dominio más amplio, como la orientación, la forma, el color, el tamaño, etc.

b) En el quale constitutivo se especifica la información relativa al todo al que pertenece o a las partes que conforman el objeto denotado, además del material del que está constituido y su peso.

c) En el quale télico se representan eventos asociados con las funciones del objeto denotado.

d) En el quale agentivo se detalla el proceso que le dio origen y los elementos involucrados en la creación de la entidad designada.

Sobre la base de esta teoría, Adelstein (2011) propone una tipología de procesos semánticos fundados en cinco criterios que contribuyen a la detección y caracterización de la neología semántica nominal mediante la determinación de la distancia semántica entre el sentido nuevo y el sentido establecido. A continuación se presenta dicha tipologización ilustrada con ejemplos de la variante chilena del español:

Tipo I: El conocimiento extralingüístico determina el sentido del neologismo semántico.

Los neologismos de este tipo no mantienen, en principio, ningún rasgo en común de la estructura de qualia del sentido establecido, sino que se fundan en el conocimiento de mundo asociado a los referentes. Por ejemplo, el sentido neológico de 'pingüino' con el significado de 'estudiante secundario que participa en protestas' no parece conservar ningún rasgo de la estructura de qualia del sentido establecido: "Ave palmípeda de unos cuatro decímetros de largo [...], con el lomo negro y el pecho y vientre blancos [...] que, por sus malas condiciones para andar y volar, se deja coger fácilmente" (Diccionario de la lengua española, 2001). El cambio semántico no sería explicable mediante el modelo del Lexicón Generativo, sino a través del conocimiento extralingüístico: 'pingüino' se refiere a 'estudiante secundario', dada la similitud del color del plumaje 
de estas aves de postura erguida con el del tradicional uniforme que visten los escolares chilenos.

Tipo II: El neologismo semántico no mantiene el subquale que lo define ("es un") del quale formal ni del quale télico.

Los neologismos de este tipo mantienen sólo una parte del quale formal, pero predicado de una manera distinta. El neologismo 'quintil', por ejemplo, que se refiere a "la quinta parte de una población estadística jerarquizada, que sirve para caracterizar la distribución del ingreso", conserva sólo parte del quale formal, el subquale que alude al número ordinal 'quinto' del sentido establecido: “quinto mes del año en el primitivo calendario romano" (Diccionario de la lengua española, 2001).

Tipo III: El neologismo semántico no mantiene el subquale "es un" del quale formal pero sí del quale télico.

A este tipo de neologismos pertenecen unidades que se basan en uno de los qualia que expresan predicación, pero los argumentos son especificados de manera diferente. En su nuevo sentido, 'taco' es un "embotellamiento o congestión de vehículos" que conserva gran parte de la estructura de qualia de dos de las acepciones de los sentidos establecidos, a saber: "montón (conjunto de cosas sin orden unas encima de otras)" y "embrollo, lío" (Diccionario de la lengua española, 2001). Sin embargo, hay una especificación diferente de los argumentos 'montón' y 'embrollo, lío', que pasa a ser un 'montón de autos', cuya circulación está entorpecida por un 'embrollo, lío' (Diccionario de la lengua española, 2001).

Tipo IV: El neologismo semántico mantiene valores de varios qualia.

Este tipo está formado por neologismos que obedecen a procesos metafóricos o metonímicos. Estos neologismos mantienen distintos tipos de rasgos, principalmente "es un" del quale formal, pero cambia el quale télico. La unidad 'rostro', en su sentido neológico, presenta varios rasgos en común con el sentido establecido: "Cara de las personas" (Diccionario de la lengua española, 2001), ya que, al igual que en el sentido registrado, el neologismo 'rostro' es un "elemento constitutivo y caracterizador de una persona", pero en el nuevo sentido hay un proceso metonímico (sinécdoque) de 'cara' por ' persona'. Se produce una nueva especificación del quale télico, ya que el nuevo sentido de 'rostro' no caracteriza a un individuo común, sino a una persona popularizada por los medios de comunicación visual. 
Tipo V: El neologismo semántico mantiene casi toda la estructura de qualia.

Este tipo comparte casi toda la estructura de qualia con el sentido no neológico, ya que se modifica un único rasgo. Se trata, en la mayoría de los casos, de procesos de especialización y, a veces, es difícil determinar si se trata de un nuevo sentido o de una especificación de la referencia. Por esta razón, los diccionarios suelen incluirlos como ejemplos o como subacepciones (Berri \& Adelstein, 2010).

A este tipo también pertenecen los neologismos formados por antonomasia, asociados a marcas registradas o a modelos de éstas: 'dockers', "pantalón de buen diseño para hombres" y'neoprén' “adhesivo económico a base de caucho natural". Aquí se produce un cambio de nombre propio a nombre común y se conserva toda la estructura de qualia. La única diferencia con el sentido establecido es que en el nuevo hay una pequeña especificación del quale agentivo asociado a su origen, puesto que el nombre propio que designa la marca de un producto pasa a designar el producto en sí.

\section{Metodología}

El análisis comprendió las unidades léxicas correspondientes a neologismos semánticos de la categoría nominal, recopilados por la Antena Chilena de Neología y alojados en el banco de datos del Observatorio de Neología (BOBNEO). La recopilación se realizó entre el 1 de enero de 2003 y el 31 de diciembre de 2014 y las unidades provinieron de dos periódicos chilenos: El Mercurio, de circulación nacional, y El Sur, de circulación regional.

De acuerdo al criterio de la Antena Chilena de Neología, se consideró la inclusión de las piezas léxicas neológicas que tuvieran cinco o más repeticiones; de esta manera, se alcanzó una muestra de 26 unidades para el presente análisis. Además, con el propósito de establecer la vigencia de estos neologismos en la variante chilena del español, se constató la fecha del primer y del último registro de cada unidad en el BOBNEO.

La verificación neológico-lexicográfica se realizó mediante el contraste en los corpus de referencia:

- Diccionario de la lengua española (DRAE actualizado). 22a. edición. Madrid: Espasa Calpe, 2001 (versión en línea). Esta versión 
electrónica permite acceder también a las enmiendas incorporadas posteriormente.

- Diccionario de uso del español de América y España (VOXUSO). Barcelona: Spes Editorial SL, 2003.

Además, la condición neológica para la variante chilena del español se verificó en las siguientes obras:

- Diccionario de uso del español de Chile (DUECh). Santiago: Editorial MN, 2010.

- Nuevo diccionario ejemplificado de chilenismos (NDECh), Félix Morales Pettorino, Valparaíso, Puntángeles, 2010.

Al cotejar las unidades con este corpus de exclusión chileno, se constató que algunas de ellas eran no neológicas para esta variante del español; sin embargo, se mantuvieron para el presente estudio por serlo para las demás variantes que conforman la Red Antenas Neológicas.

Puesto que los datos para este trabajo abarcan un período extenso, se verificó cada unidad en el corpus de exclusión DRAE en línea hasta el 31/12/2014, ya que esta obra de referencia se actualiza de manera frecuente y no así los demás diccionarios empleados. La finalidad de este procedimiento fue comprobar cuáles de estas 26 unidades habían dejado de ser neológicas durante la investigación.

La glosa para cada unidad se definió mediante el contexto lingüístico en que se habían encontrado las unidades, el corpus de exclusión de la variante chilena, información extraída de la web y el conocimiento de mundo. Para ilustrar los nuevos sentidos se utilizaron ejemplos del BOBNEO.

En caso de que una unidad tuviese más de un sentido establecido en el corpus de exclusión, se eligió la pieza léxica no neológica que tuviera mayor similitud con la nueva, con la finalidad de realizar una comparación que reflejara mejor el nuevo sentido.

\section{Resultados}

Se comprobó que todas las unidades eran lexicográficamente neológicas, a excepción de dos, cuya inclusión en este estudio obedece a su reciente sanción en el Diccionario de la lengua española en línea: 
actor, en su acepción "participante en una acción o suceso", y raqueta con el sentido de "deportista que juega con raqueta" (DRAE, 2001). Llama la atención que actor, neologismo de uso frecuente en la prensa, no haya sido recogido por ninguno de los diccionarios de chilenismos consultados para este estudio. La Tabla 1 presenta las unidades neológicas del corpus con sus definiciones y su presencia en las obras lexicográficas de referencia.

\begin{tabular}{|c|c|c|c|c|c|c|}
\hline \multirow{2}{*}{\multicolumn{2}{|c|}{$\begin{array}{l}\text { Unidad léxica } \\
\text { neológica }\end{array}$}} & \multirow{2}{*}{ Nuevo sentido } & \multicolumn{4}{|c|}{$\begin{array}{l}\text { Obras lexicográficas de } \\
\text { referencia }\end{array}$} \\
\hline & & & \multicolumn{4}{|c|}{ actualizado } \\
\hline 1. & acero & $\begin{array}{l}\text { Jugador del Club Deportivo } \\
\text { Huachipato, fundado por la } \\
\text { compañía siderúrgica del mismo } \\
\text { nombre. }\end{array}$ & --- & --- & --- & --- \\
\hline 2. & actor & $\begin{array}{l}\text { Persona que interviene en } \\
\text { actividades de la vida real. }\end{array}$ & --- & Sí & --- & --- \\
\hline 3. & azul & $\begin{array}{l}\text { "Jugador o hincha del equipo de } \\
\text { fútbol de la Universidad de Chile" } \\
\text { (DUECh, 2010:102), cuyo uniforme } \\
\text { es de color azul. }\end{array}$ & --- & --- & Sí & Sí \\
\hline 4. & barrista & $\begin{array}{l}\text { "Integrante de una barra o } \\
\text { hinchada". (DUECh, 2010:111). }\end{array}$ & --- & --- & Sí & --- \\
\hline 5. & campamento & $\begin{array}{l}\text { Barrio marginal con viviendas de } \\
\text { calidad muy precaria y de tamaño } \\
\text { reducido, que emerge de forma } \\
\text { espontánea y paulatina. }\end{array}$ & --- & --- & --- & Sí \\
\hline & carrete & $\begin{array}{l}\text { Especialmente entre los jóvenes, } \\
\text { evento de esparcimiento, } \\
\text { generalmente nocturno y con baile y } \\
\text { alcohol. (DUECh, 2010). }\end{array}$ & --- & --- & Sí & Sí \\
\hline & colorín & $\begin{array}{l}\text { Partidario de la corriente que en } \\
\text { el Partido Demócrata Cristiano } \\
\text { de Chile representaba el pelirrojo } \\
\text { Adolfo Zaldívar. }\end{array}$ & -- & --- & Sí & --- \\
\hline & cruzado & $\begin{array}{l}\text { "Jugador o hincha del equipo de } \\
\text { fútbol de la Universidad Católica } \\
\text { de Chile" (DUECh, 2010:303), cuya } \\
\text { camiseta tiene una cruz el pecho. }\end{array}$ & --- & --- & Sí & Sí \\
\hline & dupla & $\begin{array}{l}\text { Dos personas de igual o distinto } \\
\text { sexo que conforman un equipo para } \\
\text { realizar una tarea. }\end{array}$ & --- & --- & --- & Sí \\
\hline & láser/laser & $\begin{array}{l}\text { Tipo de embarcación a vela, liviana, } \\
\text { para un solo tripulante. }\end{array}$ & --- & --- & --- & Sí \\
\hline
\end{tabular}




\begin{tabular}{|c|c|c|c|c|c|c|}
\hline 11. & minero & $\begin{array}{l}\text { Jugador o hincha de los equipos de } \\
\text { fútbol Cobreloa, Cobresal, Lota- } \\
\text { Schwager y O'Higgins, de ciudades } \\
\text { vinculadas a la minería. }\end{array}$ & --- & --- & Sí & Sí \\
\hline 12. & $\operatorname{mix}$ & $\begin{array}{l}\text { Mezcla o combinación de varios } \\
\text { elementos. }\end{array}$ & --- & --- & --- & Sí \\
\hline 13. & nicho & $\begin{array}{l}\text { Espacio no físico que se forma a } \\
\text { partir de necesidades por satisfacer o } \\
\text { por despertar. }\end{array}$ & --- & --- & --- & Sí \\
\hline 14. & oncena & $\begin{array}{l}\text { Equipo de fútbol, en razón del } \\
\text { número de jugadores que lo } \\
\text { integran. }\end{array}$ & -- & --- & --- & Sí \\
\hline 15. & papayero & $\begin{array}{l}\text { "Jugador o hincha del equipo de } \\
\text { fútbol de Deportes La Serena" } \\
\text { (DUECh, 2010:655), ciudad } \\
\text { relacionada con el cultivo de } \\
\text { papayas. }\end{array}$ & -- & --- & Sí & Sí \\
\hline 16. & pilates & $\begin{array}{l}\text { Sistema de entrenamiento físico y } \\
\text { mental creado por Joseph Hubertus } \\
\text { Pilates para el control mental, la } \\
\text { respiración y la relajación. }\end{array}$ & --- & --- & --- & Sí \\
\hline 17. & pingüino & $\begin{array}{l}\text { Estudiantes de enseñanza básica } \\
\text { o media que participan en } \\
\text { manifestaciones por la reforma } \\
\text { educacional. }\end{array}$ & --- & --- & Sí & Sí \\
\hline 18. & plumavit & $\begin{array}{l}\text { Material sintético liviano de } \\
\text { poliestireno expandido, que se } \\
\text { emplea para embalaje o aislación } \\
\text { térmica. (DUECh, 2010). }\end{array}$ & -- & --- & Sí & Sí \\
\hline 19. & pokemón & $\begin{array}{l}\text { Miembro de una tribu urbana que } \\
\text { luce ropa y peinado llamativos, } \\
\text { exhibe apatía social y rememora a los } \\
\text { personajes de Pokémon }{ }^{\circledR} \text {. }\end{array}$ & --- & --- & Sí & Sí \\
\hline 20. & portafolio & $\begin{array}{l}\text { Acortamiento de 'portafolio } \\
\text { de inversiones', conjunto de } \\
\text { instrumentos financieros para } \\
\text { realizar inversiones. }\end{array}$ & --- & --- & --- & --- \\
\hline 21. & quince & $\begin{array}{l}\text { Equipo de rugby, en razón del } \\
\text { número de jugadores que lo } \\
\text { integran. }\end{array}$ & --- & --- & --- & Sí \\
\hline 22. & quintil & $\begin{array}{l}\text { Quinta parte de una población } \\
\text { estadística ordenada de menor a } \\
\text { mayor, que sirve para caracterizar la } \\
\text { distribución del ingreso. }\end{array}$ & --- & --- & --- & Sí \\
\hline 23. & raqueta & $\begin{array}{l}\text { "Deportista que juega con raqueta" } \\
\text { (DRAE en línea). }\end{array}$ & -- & Sí & --- & Sí \\
\hline 24. & rostro & $\begin{array}{l}\text { Persona muy conocida, convertida } \\
\text { en símbolo de un producto, marca o } \\
\text { canal de televisión. }\end{array}$ & --- & --- & --- & Sí \\
\hline
\end{tabular}




\begin{tabular}{|c|c|c|c|c|c|c|}
\hline & single & $\begin{array}{l}\text { Partido de tenis jugado por dos } \\
\text { oponentes individuales. }\end{array}$ & --- & --- & --- & $\square$ \\
\hline & taco & $\begin{array}{l}\text { “Atasco o atochamiento del tránsito } \\
\text { de vehículos en un lugar" (DUECh, } \\
\text { 2010:856). }\end{array}$ & --- & --- & $\square$ & $\square$ \\
\hline
\end{tabular}

Tabla 1. Unidades del corpus con sus definiciones y cotejo en los diccionarios

El NDECh recoge la mayoría de las unidades (21) y el DUECh sólo 11, pero dos de ellas, barrista y colorín, no se encuentran en el primer diccionario. Esto demuestra que casi el $90 \%$ de las unidades en estudio no son neológicas para el español de Chile. Su presencia en los diccionarios regionales reflejaría su carácter testimonial, ya que corresponden a referentes típicamente chilenos, o bien que se denominan de manera diferente en esta variante o que son importantes para el hablante chileno.

Por otra parte, se constató la vigencia de las unidades en el BOBNEO (ver Tabla 2).

\begin{tabular}{rlcr}
\hline \multicolumn{2}{l}{ Unidad léxica neológica } & $\begin{array}{c}\text { Período de registro } \\
\text { (BOBNEO) }\end{array}$ & Años \\
\hline 1. & acero & $2006-2014$ & 9 \\
\hline 2. & actor & $2006-2014$ & 9 \\
\hline 3. & azul & $2004-2014$ & 11 \\
\hline 4. & barrista & $2003-2012$ & 12 \\
\hline 5. & campamento & $2005-2014$ & 10 \\
\hline 6. & carrete & $2004-2014$ & 11 \\
\hline 7. & colorín & $2005-2009$ & 5 \\
\hline 8. & cruzado & $2004-2013$ & 10 \\
\hline 9. & dupla & $2006-2011$ & 6 \\
\hline 10. & láser/laser & $2004-2006$ & 3 \\
\hline 11. & minero & $2004-2014$ & 11 \\
\hline 12. & mix & $2005-2014$ & 10 \\
\hline 13. & nicho & $2003-2014$ & 12 \\
\hline 14. & oncena & $2004-2014$ & 11 \\
\hline 15. & papayero & $2004-2014$ & 11 \\
\hline 16. & pilates & $2006-2014$ & 9 \\
\hline 17. & pingüino & $2006-2014$ & 9 \\
\hline & & & \\
\hline
\end{tabular}




\begin{tabular}{rlrr}
\hline 18. & plumavit & $2005-2012$ & 8 \\
\hline 19. & pokemón & $2008-2011$ & 4 \\
\hline 20. & portafolio & $2003-2014$ & 12 \\
\hline 21. & quince & $2004-2009$ & 6 \\
\hline 22. & quintil & $2005-2014$ & 10 \\
\hline 23. & raqueta & $2006-2014$ & 9 \\
\hline 24. & rostro & $2006-2014$ & 9 \\
\hline 25. & single & $2004-2009$ & 6 \\
\hline 26. & taco & $2003-2014$ & 12 \\
\hline
\end{tabular}

Tabla 2. Vigencia de las unidades neológicas en el BOBNEO

Como se observa en la Tabla 2, la mayoría de las unidades del corpus demostró vigencia en la prensa hasta el final de esta investigación. Sin embargo, algunas tuvieron corta duración en la prensa, lo que implicaría que el referente dejó de consignarse, ya sea por cambios en el acontecer nacional o internacional o por sesgo de la prensa o del pesquisidor de neologismos. Por ejemplo, deportes que en algún momento hicieron noticia, por alguna razón han dejado de tener prominencia (dupla, láser, quince, single). También podría aducirse que las piezas léxicas podrían haber caído en desuso al desaparecer los referentes de la contingencia (colorín, pokemón).

El siguiente análisis presenta la tipologización de las unidades léxicas neológicas en función de la distancia semántica entre el sentido nuevo y el establecido, basada en el trabajo de Berri y Adelstein (2010), que a su vez se funda en la Teoría del Lexicón Generativo de Pustejovsky (1991, 1995). El estudio aborda los siguientes aspectos de tipificación: la información compartida entre el sentido establecido y el nuevo, la naturaleza del cambio semántico que se produce en cada tipo y la forma en que se configura el significado.

Al Tipo I corresponden las unidades que poseen la máxima distancia semántica con respecto al sentido establecido, porque la unidad nueva no comparte ninguna información con la no neológica, y el sentido se puede desentrañar sólo a partir del contexto y del conocimiento de mundo de los hablantes. Al no tener las unidades ningún rasgo semántico en común, el cambio semántico no es explicable por la Lingüística Generativa y, en consecuencia, el nuevo significado es completamente diferente de aquel 
del referente original. Si bien los dos sentidos no comparten, en principio, ningún valor de la estructura de qualia, se podría inferir que existe una información mínima compartida en algún subquale (ver Tabla 3).

\begin{tabular}{cccc}
\hline Entrada & $\begin{array}{c}\text { Información } \\
\text { compartida }\end{array}$ & Distancia semántica & Configuración \\
\hline $\begin{array}{c}\text { colorín, carrete, } \\
\text { pingüino }\end{array}$ & Ninguna & Máxima & Nuevo significado \\
\hline
\end{tabular}

Tabla 3. Caracterización de las unidades neológicas del Tipo I

\section{Ejemplo: pingüino}

En su sentido establecido corresponde a un 'ave palmípeda que camina erguida, de lomo negro y pecho y vientre blancos', mientras que en su sentido neológico se refiere principalmente a los 'estudiantes secundarios que participan en manifestaciones por la reforma educacional’. La nueva connotación surgiría por analogía con el uniforme que visten los escolares en Chile: una chaqueta azul oscuro y una camisa blanca. Se podría inferir que lo que determina el nuevo sentido es la metáfora que surge del subquale 'color' por la similitud en la distribución de colores en ambos referentes.

Es interesante destacar la diferencia del sentido neológico entre el pingüino acuñado en Chile y el empleado en Argentina ('seguidores de Kirchner'), lo que demuestra que este tipo de neologismo sólo es explicable a través del contexto y del conocimiento extralingüístico y no mediante la estructura de qualia.

Los pingüinos, como se les llamó a los estudiantes, lograron poner en la agenda pública el tema de la calidad de la educación y la reforma a la Ley Orgánica Constitucional de Enseñanza. [El Sur, 19/11/2006]

Al Tipo II pertenece una gran variedad de neologismos y en todos ellos la distancia semántica es mayor. El sentido de la unidad neológica mantiene uno o más valores de un mismo quale del sentido establecido, pero no conserva la información completa de ese quale. La información compartida puede corresponder a los qualia constitutivo, formal, agentivo o télico. El tipo de cambio semántico correspondería tradicionalmente a 
diversos procesos metafóricos o metonímicos. La Tabla 4 da cuenta de la información compartida y no compartida entre la unidad establecida y la neológica para cada entrada.

\begin{tabular}{|c|c|}
\hline Entrada & Información compartida \\
\hline acero & $\begin{array}{l}\text { Comparte con el sentido establecido el valor de un subquale: el } \\
\text { agentivo (origen). Se diferencia del sentido establecido en los valores } \\
\text { del quale télico y del formal: ya no "es un". }\end{array}$ \\
\hline azul & $\begin{array}{l}\text { Comparte con el sentido establecido el valor de un subquale del quale } \\
\text { formal: color. Se diferencia en los valores del quale télico y del formal: } \\
\text { ya no "es un". }\end{array}$ \\
\hline barrista & $\begin{array}{l}\text { El cambio semántico se produjo en 'barra'. Cambian los valores del } \\
\text { quale télico y los del quale formal: "ya no es un”. }\end{array}$ \\
\hline cruzado & $\begin{array}{l}\text { Comparte con el sentido establecido el valor de un subquale del } \\
\text { quale formal: la cruz. Se diferencia en los valores del quale télico y del } \\
\text { formal: ya no "es un". }\end{array}$ \\
\hline dupla & $\begin{array}{l}\text { Comparte con el sentido establecido el valor de un subquale } \\
\text { constitutivo: el número dos. Se diferencia en los valores del quale } \\
\text { télico y del formal: ya no "es un". }\end{array}$ \\
\hline láser/laser & $\begin{array}{l}\text { Comparte con el sentido establecido valores de subqualia del quale } \\
\text { formal: tránsito veloz, direccional, angosto. Se diferencia en el quale } \\
\text { télico y en el quale formal "es un". }\end{array}$ \\
\hline minero & $\begin{array}{l}\text { Comparte con el sentido establecido el valor de un subquale: el } \\
\text { agentivo (origen). Se diferencia en los valores del quale télico y del } \\
\text { formal: ya no "es un". }\end{array}$ \\
\hline oncena & $\begin{array}{l}\text { Comparte con el sentido establecido el valor de un subquale } \\
\text { constitutivo: formado por once elementos. Se diferencia en los valores } \\
\text { del quale télico y del formal: ya no "es un". }\end{array}$ \\
\hline papayero & $\begin{array}{l}\text { Comparte con el sentido establecido el valor de un subquale: el } \\
\text { agentivo (origen). Se diferencia en los valores del quale télico y del } \\
\text { formal: ya no "es un". }\end{array}$ \\
\hline pilates & $\begin{array}{l}\text { Comparte con el sentido establecido un valor de un subquale del } \\
\text { quale télico, pero hay un deslizamiento de información del quale télico } \\
\text { al agentivo. Se diferencia en los valores del quale formal: ya no "es } \\
\text { un". }\end{array}$ \\
\hline pokemón & $\begin{array}{l}\text { Comparte con el sentido establecido valores de un subquale del quale } \\
\text { formal: apariencia física. Se diferencia en el quale formal "es un" y en } \\
\text { el quale télico. }\end{array}$ \\
\hline quince & $\begin{array}{l}\text { Comparte con el sentido establecido el valor de un subquale } \\
\text { constitutivo: formado por quince elementos. Se diferencia en los } \\
\text { valores del quale télico y del formal: ya no "es un". }\end{array}$ \\
\hline quintil & $\begin{array}{l}\text { Comparte con el sentido establecido el valor de un subquale del quale } \\
\text { formal: 'quinto lugar'. Se diferencia en los valores del quale télico y } \\
\text { del formal: ya no "es un". }\end{array}$ \\
\hline
\end{tabular}




\begin{tabular}{ll}
\hline raqueta & $\begin{array}{l}\text { Comparte con el sentido establecido un subquale del quale télico: } \\
\text { jugar, pero se especifica de manera distinta. Se diferencia en el quale } \\
\text { télico y en el formal: ya no "es un". }\end{array}$ \\
\hline single & $\begin{array}{l}\text { Comparte con el sentido establecido el valor de un subquale } \\
\text { constitutivo: formado por un solo elemento. Se diferencia en los } \\
\text { valores del quale télico y del formal: ya no "es un". }\end{array}$ \\
\hline
\end{tabular}

Tabla 4. Caracterización de las unidades neológicas del Tipo II

\section{Ejemplo: quintil}

Quintil, en el sentido no neológico, es el 'quinto mes del año en el primitivo calendario romano', mientras que en el sentido nuevo se refiere a 'la quinta parte de una población estadística, que sirve para caracterizar la distribución del ingreso'. En quintil, el sentido establecido y el neológico comparten solamente una parte del quale formal, que se refiere al ordinal 'quinto'. Sin embargo, no comparten el subquale del quale formal 'es un', ya que uno es un 'mes' y el otro, una 'población estadística'.

Explicó que el Estado no ha enfrentado el tema del ingreso progresivo de los quintiles más pobres a la educación superior, considerando que la ampliación de la cobertura se da entre los grupos de menores ingresos. [El Sur, 17/04/2005]

En el tipo III, la distancia semántica entre el sentido neológico y el establecido es menor, ya que la información compartida se refleja en una cantidad importante de rasgos semánticos, sobre todo en los argumentos de los qualia que más aportan al significado.

\section{Ejemplo: portafolio}

En portafolio hay una nueva especificación de los argumentos del quale télico 'contener', que se actualiza de manera diferente: de 'contener papeles' pasa a 'contener instrumentos financieros'. Los neologismos de este grupo comparten valores de uno o más qualia, pero sus argumentos se especifican de manera distinta en el sentido neológico respecto del sentido establecido.

Los factores que determinaron esa inversión fueron la convicción del gran potencial vitivinícola de ese país y la complementariedad 
del proyecto que incorporaba nuevos orígenes y cepas distintivas al portafolio global. [El Mercurio, 12/12/2003]

En el Tipo IV (ver Tabla 5), la distancia semántica entre el sentido establecido y el nuevo es pequeña, ya que los neologismos de este tipo comparten muchos rasgos semánticos de la estructura de qualia, especialmente el subquale del quale formal 'es un', pero presentan nuevos valores en el quale télico. El nuevo sentido surge como consecuencia de esta nueva configuración semántica.

\begin{tabular}{ll}
\hline Entrada & Información compartida \\
\hline actor & $\begin{array}{l}\text { Comparte casi toda la estructura de qualia, pero hay una nueva } \\
\text { especificación de los argumentos del quale télico. }\end{array}$ \\
\hline campamento & $\begin{array}{l}\text { Comparte casi toda la estructura de qualia, pero hay una nueva } \\
\text { especificación de los argumentos de subqualia de los qualia formal } \\
\text { y télico. }\end{array}$ \\
\hline mix & $\begin{array}{l}\text { Comparte casi toda la estructura de qualia, pero hay una nueva } \\
\text { especificación de los argumentos del quale constitutivo. }\end{array}$ \\
\hline nicho & $\begin{array}{l}\text { Comparte con el sentido establecido el quale formal 'es un' y parte } \\
\text { del quale télico, pero éste tiene una nueva especificación: 'llenar } \\
\text { con cosas' vs. 'llenar con ideas o propuestas'. }\end{array}$ \\
\hline rostro & $\begin{array}{l}\text { Comparte casi toda la estructura de qualia, pero cambia el quale } \\
\text { constitutivo. }\end{array}$ \\
\hline taco & $\begin{array}{l}\text { Comparte con el sentido establecido valores del quale formal: "es un", } \\
\text { pero hay una nueva especificación de los argumentos del quale télico: }\end{array}$ \\
\hline
\end{tabular}

Tabla 5. Caracterización de las unidades neológicas del Tipo IV

\section{Ejemplo: nicho}

La unidad neológica nicho, con el sentido de 'espacio abstracto que se forma a partir de necesidades por satisfacer o por despertar', mantiene parte de los valores del quale formal, sobre todo el subquale 'es un' (concavidad), que se distingue del sentido establecido en que la concavidad se llena con intangibles. Además, mantiene parte del quale télico (llenar): en el sentido neológico se 'llena con ideas para satisfacer necesidades', mientras que en el no neológico, 'se llena con cuerpos u objetos'.

Falta creatividad y un poco de audacia, porque indudablemente requiere tener una audacia importante descubrir cuáles son esos nichos del mercado que no están explotados todavía. [El Mercurio, $12 / 05 / 2007]$ 
En el tipo V, el nuevo sentido comparte prácticamente toda la estructura de qualia con el sentido establecido, en especial el subquale del quale formal 'es un' y el quale télico, por lo que la distancia semántica es mínima. El nuevo sentido cambia en un solo valor, que no es siempre el mismo. Desde la óptica tradicional, a este cambio semántico corresponden las unidades creadas por extensión, que se incorporarían a los diccionarios como subacepciones de una entrada o ejemplos de ella. Además, a este tipo pertenecen también las unidades neológicas creadas por antonomasia, como las marcas registradas.

\section{Ejemplo: plumavit}

Los sentidos neológico (plumavit) y no neológico (Plumavit) comparten toda la estructura de qualia, pero hay una leve especificación del quale agentivo.

Para aislar el techo y las paredes se puede usar una infinidad de aislantes disponibles en el mercado, pero Veas indica que los dos materiales más fáciles de encontrar y a menor costo son el poliestireno expandido, conocido como plumavit, y la lana mineral, también llamado 'aislan'. [El Mercurio, 01 / 05 / 2010]

A la luz del análisis de las piezas léxicas que componen el corpus, se estableció una adecuación de las distintas brechas de la distancia semántica entre el sentido establecido y el nuevo. En efecto, se definió una nueva gradación de esta distancia semántica sobre la base del trabajo de Berri y Adelstein (2010), con el propósito de afinar la clasificación de la neología semántica propuesta por estas autoras. En la presente definición se ha establecido la categoría 'distancia máxima' para clasificar las piezas neológicas que no presentaban ninguna información compartida con la unidad léxica establecida, por lo que no resultaba posible explicar el nuevo sentido a través de la Teoría de Qualia.

Por otra parte, se reetiquetó la categoría 'muy pequeña' (Berri \& Adelstein, 2010) como 'mínima' en contraposición con la categoría 'máxima' aquí propuesta. La siguiente tabla muestra la correlación entre las definiciones de la tipología de Berri y Adelstein (2010) y las sugeridas en este trabajo. El propósito ha sido establecer una categoría bien definida para cada tipo de neologismo semántico, de acuerdo a la información 
compartida en la estructura de qualia entre los sentidos establecido y nuevo (ver Tabla 6).

\begin{tabular}{llcc}
\hline Tipo & \multicolumn{1}{c}{ Información compartida } & $\begin{array}{c}\text { Distancia semántica } \\
\text { (Berri \& Adelstein, } \\
\text { 2010) }\end{array}$ & $\begin{array}{c}\text { Distancia } \\
\text { semántica } \\
\text { propuesta }\end{array}$ \\
\hline I & $\begin{array}{l}\text { Cero. No explicable por la Teoría de } \\
\text { Qualia }\end{array}$ & Mayor & Máxima \\
\hline II & Uno o más valores de un mismo quale & Mayor & Mayor \\
\hline III & $\begin{array}{l}\text { Varios argumentos de qualia que más } \\
\text { aportan al significado }\end{array}$ & Menor & Menor \\
\hline IV & Varios qualia & Pequeña & Pequeña \\
\hline V & $\begin{array}{l}\text { Prácticamente toda la estructura de } \\
\text { qualia }\end{array}$ & Muy pequeña & Mínima \\
\hline
\end{tabular}

Tabla 6. Correlación entre las categorizaciones de Berri y Adelstein y la propuesta

\section{Conclusión}

Este estudio permitió verificar la aplicabilidad de la Teoría de Qualia en el corpus chileno al comparar las piezas léxicas neológicas con las correspondientes piezas no neológicas que presentaban la menor distancia semántica en las obras lexicográficas de exclusión. De este modo, al emplear rótulos más definidos, se lograría una clasificación más clara de los distintos tipos de neologismos semánticos. Esto tendría consecuencias prácticas para neólogos, lexicólogos y lexicógrafos, por cuanto se dispondría de un respaldo teórico que facilitaría la tarea de descripción, clasificación, definición o sanción de los neologismos semánticos. Así, el 'sentimiento de neologicidad' (Sablayrolles, 2000) asociado a la intuición no sería el elemento decisivo en el análisis de los neologismos semánticos.

Para el Tipo I, en el que no existe distancia semántica, porque no hay información compartida en la estructura de qualia, el contexto lingüístico opera como elemento determinante para establecer la neologicidad. Nazar y Vidal (2008) plantean que la aparición sistemática de una unidad léxica en un contexto distinto al habitual indica que se está en presencia de un neologismo. Si bien el contexto lingüístico tiene importancia en la detección de neologismos semánticos, es a través de la actualización u operación interpretativa que se puede construir el nuevo sentido en su totalidad. 
La Teoría de Qualia es aplicable a la descripción de la distancia semántica de los neologismos de los tipos II al V. La distancia semántica que caracteriza a cada tipo se mide en función de la información compartida en la estructura de qualia entre el sentido establecido y el nuevo. A menor cantidad de información compartida entre ambos sentidos, mayor distancia semántica y, por ende, el cambio semántico también es mayor. Cuanto más precisa sea la gradación de la distancia semántica entre un sentido y otro, más exhaustivo será el análisis de la neologicidad y más objetivable la sanción de las nuevas piezas léxicas. 


\section{Bibliografía}

Academia Chilena de la Lengua. (2010). Diccionario de uso del español de Chile. Santiago: MN Editorial Ltda.

Adelstein, A. (2011). Metodología de trabajo neológico y tipologías: aspectos de la neología semántica. Actas del II Congreso Internacional de Neología de las Lenguas Románicas, Sao Paulo, Brasil.

Álvarez de Mon Rego, I. \& Álvarez-Bolado Sánchez, C. (2013). Semantic neology in the domain of videogames in Spanish. Ibérica, 25, 63-84.

Battaner, M.P. (2002). Diccionario de uso del español de América y España. (1ra. Ed.). Barcelona: Spes Editorial.

Berri, M. (2014). Polisemia regular y piezas léxicas en contexto. En A. Adelstein, Interfaces semánticas (pp. 45-58). Mendoza: Editorial de la Facultad de Filosofía y Letras de la Universidad Nacional de Cuyo.

Berri, M. \& Adelstein, A. (2010). Hacia una tipología de la neología semántica nominal. En V. Castel \& L. Cubo de Severino (Eds.). La renovación de la palabra en el bicentenario de la Argentina. Los colores de la mirada lingüística (pp. 213-221). Mendoza: Editorial de la Facultad de Filosofía y Letras de la Universidad Nacional de Cuyo.

Cabré, M. T. (2006). La clasificación de neologismos: una tarea compleja. Alfa, 50(2), 229-250.

Cabré, M. T. (2009). La classificació dels neologismes: una tasca complexa. En M.T. Cabré \& R. Estopà (Eds.). Les paraules noves: criteris per detectar i mesurar els neologismes (pp. 1137). Barcelona: Eumo; Universitat Pompeu Fabra.

Cabré, M. T., Bayà, M.R., Bernal, E., Freixá, J., Solé, E. \& Vallès, T. (2002). Evaluación de la vitalidad de una lengua a través de la neología: a propósito de la neología espontánea y de la neología planificada. En Lexic $i$ Neologia (pp.159-201). Barcelona: Observatori de Neologia. 
Institut Universitari de Lingüística Aplicada, Universitat Pompeu Fabra,

Cabré Castelví, M. T. \& Estopà Bagot, R. (2009). Trabajar en neología con un entorno integrado en línea: la estación de trabajo OBNEO. Revista de Investigación Lingüística, 12, 1738 .

Coșeriu, E. (1973). Determinación y entorno. Dos problemas de una lingüística del habla. En Teoría del lenguaje y lingüística general (pp. 282-323). Madrid: Gredos.

De Miguel, E. (2009). La teoría del lexicón generativo. En Panorama de la Lexicología (pp. 337-368). Barcelona: Ariel.

Pequeño glosario de semántica. Universidad Nacional de Cuyo. Recuperado http: / /www.revue-texto.net/Reperes/ Glossaires/Glossaire_sp.html

Guerrero Ramos, G. (2010). Neologismos en el español actual. (3a. Ed.) Madrid: Arco Libros, SL.

Miller, G., Beckwith, R., Fellbaum, C., Gross, D. \& Miller, K. (1990). WordNet: An online lexical database. International Journal of Lexicography 3(4), 235-344.

Morales Pettorino, F. (2010). Nuevo diccionario ejemplificado de chilenismos (NDECh) Valparaíso: Puntángeles,

Nazar, R. \& Vidal, V. (2008). Aproximación cuantitativa a la neología. Actas del I Congreso Internacional de Neología de las Lenguas Románicas, Barcelona, España.

Observatori de Neología, Institut Universitari de Lingüística Aplicada, Universitat Pompeu Fabra. (2003). Recuperado de http:/ / www.iula.upf.edu/obneo/obpreses.htm

Protocolo de vaciado de prensa escrita. (2003). Observatori de Neología, Institut Universitari de Lingüística Aplicada, Universitat Pompeu Fabra (mimeo).

Pustejovsky, J. (1991). The Generative Lexicon. Computational Linguistics, 17, 409-441. 
Pustejovsky, J. (1995). The Generative Lexicon. Cambridge: MIT Press.

Pustejovsky, J. (2011). Coercion in a general theory of argument selection. Linguistics, 49(6), 1401-1431.

Real Academia Española. (2001). Diccionario de la lengua española (22 ${ }^{a}$ ed.). Madrid: Espasa-Calpe. Recuperado de http:/ / www.rae.es/rae.html

Rey, A. (1976). Néologisme: un pseudo-concept? Cahiers de lexicologie, $28,3-17$.

Sablayrolles, J. F. (2000). La Néologie en Français Contemporain: Examen du concept et analyse de production néologiques récen. Paris: Honoré Champion. 